\title{
Coefficient identification in PDEs applied to image inpainting
}

Fredrik Berntsson and George Baravdish

\section{Linköping University Post Print}

\section{Tweet}

N.B.: When citing this work, cite the original article.

Original Publication:

Fredrik Berntsson and George Baravdish, Coefficient identification in PDEs applied to image inpainting, 2014, Applied Mathematics and Computation, (242), 227-235.

http://dx.doi.org/10.1016/j.amc.2014.05.051

Copyright: Elsevier

http://www.elsevier.com/

Postprint available at: Linköping University Electronic Press

http://urn.kb.se/resolve?urn=urn:nbn:se:liu:diva-110691 


\title{
Coefficient Identification in PDEs applied to Image Inpainting
}

\author{
Fredrik Berntsson ${ }^{\mathrm{a}}$, George Baravdish ${ }^{\mathrm{b}}$ \\ ${ }^{a}$ Linköping University, S-581 83, Linköping Sweden \\ ${ }^{b}$ Campus Norrköping, Linköping University, S-581 83, Norrköping Sweden
}

\begin{abstract}
In this paper, we introduce the concept of parameter identification problems, which are inverse problems, as a methodology to inpainting. More specifically, as a first study in this new direction, we generalize the method of harmonic inpainting by studying an elliptic equation in divergence form where we assume that the diffusion coefficient is unknown. As a first step, this unknown coefficient is determined from the information obtained by the known data in the image. Next, we fill in the region with missing data by solving an elliptic equation in divergence form using this obtained diffusion coefficient. An error analysis shows that this approach is promising and our numerical experiments produces better results than the harmonic inpainting.

Keywords: Image Inpainting, Inverse problems, Coefficient Identification
\end{abstract}

\section{Introduction}

Image inpainting is the process of filling in missing or damaged regions in images such as paintings, photographs and films. In art, inpainting of degraded paintings has traditionally been done by professional artists. In mathematics, inpainting is an interpolation problem where the basic idea is to fill-in the damaged regions by a propagation of available information from their surroundings in the image $[1,2,3]$. Image inpainting has a wide range of important applications in image processing. For instance, to remove and add objects in images, image coding and wireless image transmission.

Email addresses: fredrik.berntsson@liu.se (Fredrik Berntsson), george.baravdish@liu.se (George Baravdish) 


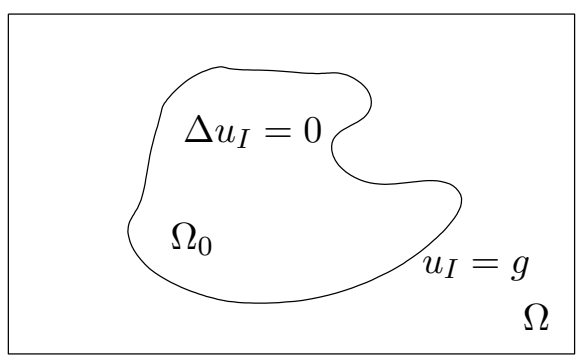

Figure 1: A rectangular image with an unknown region $\Omega_{0}$. Inpainting using the Harmonic interpolant.

In the past few years, several different approaches have been proposed to tackle this complicated image processing task. We mention the early work of Nitzberg, et al [4], on outlines of objects for image segmentation and depth extraction. This idea was extended to level-sets of images by Masnou and Morel [5]. Later on Bertalmio, et al [6], proposed an approach which came to be known as digital inpainting. This fundamental work inspired many of the forthcoming research papers on inpainting. In their approach, they formulated a third order nonlinear partial differential equation (PDE) that propagates information in the direction of the isophotes (edges). This work had a tremendous influence on the field by using PDE based mathematical methods to do digital inpainting. Some of the PDE models to mention are Navier-Stokes equation and fluid dynamics related methods [7], transport equation, Cahn-Hilliard equation [8], and Ginzburg-Landau equation [9]. Further, Variational Exemplar-based inpainting methods have recently been studied $[10,11,12]$.

We also mention the approach of minimizing energy functionals involving the bounded variation (BV) image model. The minimizer of the functionals satisfies a total variation (TV) inpainting model [13]. These functionals have later been modified to involve the curvature in the image called Euler elastica. Chan, et al [14, 15], showed, by a new technique called curvature-driven diffusion (CDD), how Euler elastica takes in consideration both curvature and transportation inpainting. Other PDE based approaches have been studied in, e.g., [16], and also in [17]. In [18], ideas from PDE-based methods were combined with the edge-reserving techniques for sharpening of edges.

Image inpainting based on diffusion type equations have been studied previously, see e.g. [19] and [20], for recent inpainting schemes based on a 
anisotropic diffusion models. Typically the coefficients of the diffusion model are estimated locally; and the resulting inpainting schemes are solved iteratively. This is in contrast to our method where the diffusion coefficient is estimated first using only known pixel values in an area surrounding the inpainting domain; see Figure 3 where $\Omega_{0}$ is the inpainting domain and the surrounding region $\Omega_{1}$ is used to estimate the diffusion coefficient. The inpainting step is then done by solving a linear differential equation, and thus no iteration is needed.

This work has been motivated by harmonic inpainting [21]. First we briefly explain the harmonic inpainting technique. Suppose $u$ is defined on a domain $\Omega \subset \mathbb{R}^{2}$ (the image) and that $u$ is unknown in a region $\Omega_{0} \subset \Omega$. The Harmonic interpolant $u_{I}$ is obtained by solving:

$$
\begin{cases}\Delta u_{I}=0, & \text { in } \Omega_{0}, \\ u_{I}=g, & \text { on } \partial \Omega_{0},\end{cases}
$$

where $\partial \Omega_{0}$ denotes the boundary of the domain $\Omega_{0}$ and $g=\left.u\right|_{\partial \Omega_{0}}$ is known. The situation is illustrated in Figure 1. The harmonic interpolant $u_{I}$ is obtained by solving a linear system of equations of dimension equal to the number of unknown pixels in the inpainting domain. The method is simple and has the advantage of being straightforward to implement and not very computationally demanding.

The simple Harmonic inpainting technique fails to reconstruct images that have sharp features. Typically in order to reconstruct images with discontinuities a non-linear equation is used; such as in the total variation inpainting scheme[13]. Our aim is to find an inpainting model that has all the advantages of Harmonic inpainting; but still does a fair job at reconstructing sharp edges inside the inpainting domain. For this purpose we turn to the equation,

$$
-\nabla \cdot\left(q \nabla u_{c}\right)=f, \quad \text { in } \quad \Omega_{0} .
$$

where the diffusion coefficient $q$ can be used to "create" sharp features inside the domain as seen in Figure 2. In the experiment the original image consists of one horizontal stripe and the inpainting domain is a square. The Harmonic interpolant $u_{I}$ fails to reconstruct the edges. For the coefficient based inpainting $u_{c}$ the coefficient $q$ was chosen so that $q=1$; except for a thin region near the edges of the original image where $q=0.01$. The result is good and the edges are reconstructed well even though the differential equation is linear. The good results can be explained as follows: Suppose the 


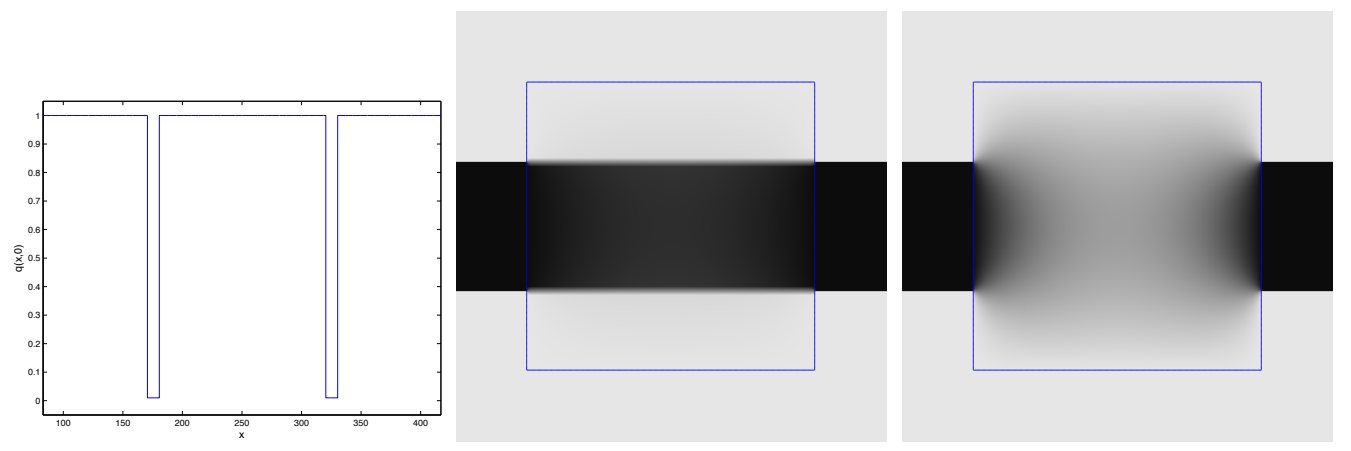

Figure 2: The coefficient $q(x, y):=q(x, 0)$ used for the motivating example (left). Also we display the results obtained using coefficient based inpainting (middle) and, for comparison, the results obtained using Harmonic inpainting (right). The coefficient $q$ was chosen "ad hoc" in order to demonstrate the potential of the method. The interior of the blue rectangle represents the domain $\Omega_{0} \cup \Omega_{1}$. For this experiment we used the source term $f=0$.

coefficient $q$ has a very low value along a line $\Gamma$ in the interior of the domain. The flow across the curve $\Gamma$ can be written $n \cdot q \nabla u$. Thus by using a small value for $q$ on $\Gamma$ we effectively split the domain into two parts and the have insulated boundary conditions $n \cdot \nabla u=0$ on the new boundary $\Gamma$. Hence, the low value for $q$ along the horizontal lines leads to a division of the inpainting domain and the result is different colored areas with a fairly sharp edge in between. Note that we do not use $q=0$ along the horizontal lines, since in that case we would not actually have a valid partial differential equation at the corresponding pixels; and would not get any results for these pixels. For this experiment the source term was $f=0$; which is resonable since the true image does not contain any local maxima or minima. However a non-zero source term $f$ is needed if we are to have local maxima or minima inside the inpainting domain. This is because the solutions of elliptic equations satisfy a maximum principle. The result indicate that an inpainting scheme using coefficients $(q, f)$ can reconstruct edges even though the equation is linear. Although a careful choice for the coefficient $q$ is necessary.

As said above, our aim is to inpaint the domain $\Omega_{0}$ by the surrounding information. Our approach is to find a diffusion coefficient $q$ and a source term $f$ such that the equation

$$
-\nabla \cdot(q \nabla u)=f, \quad \text { in } \Omega_{1},
$$




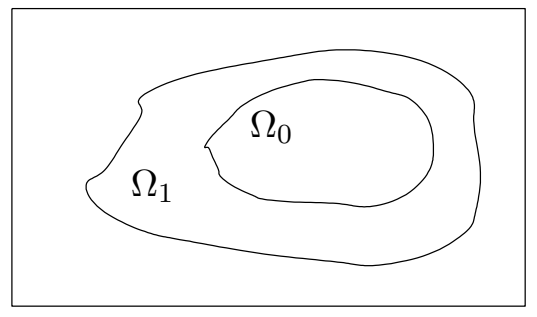

Figure 3: The image is assumed to be unknown inside the inpainting domain $\Omega_{0}$. The region $\Omega_{1}$, where the image is assumed to be known, is used for determining the appropriate diffusivity $q(x, y)$ and source term $f(x, y)$ to use in the image model.

is satisfied, where $\Omega_{1}$ is the domain surrounding the inpainting domain, as seen in Figure 3. The inverse problem (3) is ill-posed due to several reasons. Since differentiating the given data $u$ in $\Omega_{1}$ is an ill-posed problem, the solutions $q$ and $f$ do not depend continuously on the data $u$. Another reason is that the problem (3) suffers from the lack of uniqueness of identifying the parameters $q$ and $f$.

The paper is organized as follows. In Section 2 we explain the detailed mathematical principles, discretization, and implementation details, for our proposed method. Numerical experiments are presented in Section 3 and finally comparisons with other methods are discussed in Section 4.

\section{Coefficient Identification Based Inpainting}

Assume that the image $u(x, y)$ can be modeled as,

$$
-\nabla \cdot(q \nabla u)=f, \quad \text { in } \quad \Omega=\Omega_{0} \cup \Omega_{1},
$$

where the diffusivity $q(x, y)$ and the source term $f(x, y)$ depends on the individual image. The domains are illustrated in Figure 3. The inpainting domain, where the image $u(x, y)$ is unknown, corresponds to the domain $\Omega_{0}$. Our aim is to use the image information available in the region $\Omega_{1}$ to find a good image model, i.e. the functions $q(x, y)$ and $f(x, y)$, and then use the identified model for the inpainting.

In order to find a good image model we select a set of $N$ basis functions $\left\{s_{j}(x, y)\right\}_{j=1}^{N}$ defined on the set $\Omega$ and write,

$$
q(x, y)=\sum_{j=1}^{N} c_{j} s_{j}(x, y), \text { and, } f(x, y)=\sum_{j=1}^{N} d_{j} s_{j}(x, y) .
$$


We find the image model by solving the minimization problem,

$$
\min _{c_{1}=1} J(c, d), \quad J(c, d)=\|\nabla \cdot(q \nabla u)+f\|_{L^{2}\left(\Omega_{1}\right)},
$$

where $c=\left\{c_{j}\right\}$ and $d=\left\{d_{j}\right\}$. The condition $c_{1}=1$ ensures that the resulting linear least squares problem has a non-trivial solution. We cannot always expect a unique solution as demonstrated by the example presented in Figure 4. For our method to work well it is required that $q(x, y)>0$. The condition $q(x, y)=c>0$ ensures that the differential operator $\nabla \cdot q \nabla u$ is strictly elliptic so that Equation 2 has a unique solution in $L^{2}\left(\Omega_{0}\right)$ [22]. If the least squares solution $q(x, y)$ isn't positive we add a constant.

The choice of the basis functions $\left\{s_{j}(x, y)\right\}$ determines the accuracy and efficiency of our method. Since our starting point is Harmonic inpainting it is natural to select $s_{j}(x, y)$ to be eigenfunctions of the Laplace operator; with suitable boundary conditions. Thus, in our experiments, we mostly use a truncated Fourier-Sine series for representing $q(x, y)$ and $f(x, y)$. Other types of basis functions, e.g. polynomials or splines, can also be used. This is something that we intend to explore further.

Remark 2.1 The least squares problem (6) is discretized as a matrix equation of size $N_{1} \times(2 N-1)$, where $N_{1}$ is the number of pixels in the region $\Omega_{1}$. The elements of the matrix are obtained by discretizing the operators $\nabla\left(s_{j}\left(x_{k}, x_{k}\right) \nabla u\left(x_{k}, x_{k}\right)\right)$, where $u\left(x_{k}, y_{k}\right)$ is known, for each pixel $\left(x_{k}, y_{k}\right)$ inside the region $\Omega_{1}$. For the discretization we use a standard 5-point approximation of the Laplacian; where all first order derivatives are approximated using centered differences with step size $\Delta x / 2$ and thus the coefficient $q$ needs to be evaluated at half-index grid points.

Remark 2.2 The Fourier-Sine basis functions can be seen as a resonable "standard" basis set. Thus the parameters of our method are the inpainting masks for the two domains $\Omega_{0}$ and $\Omega_{1}$, and also the number of basis functions $N$ to use. Other options for basis functions are polynomials, e.g. $\{x y,(1-$ $x) y, x(1-y),(1-x)(1-y)\}$ for the linear case or similarly for quadratic or cubic polynomials. Additionally we include tests using $B$-splines as a basis set in this paper. The choice of the basis functions is an area that should be explored further.

\subsection{Error Analysis}

In this section, we study the error obtained by filling the missing part of the image by using an elliptic equation given in divergence form. Since this 
is a generalization of the harmonic inpainting, we follow the error analysis studied in [23]

Let $z_{0}=\left(x_{0}, y_{0}\right) \in \Omega_{0}$. For every $z=(x, y) \in \Omega_{0}$, we let $G\left(z_{0}, z\right)$ be the Greens function for the Poisson equation on $\Omega_{0}$, that is $G$ is the solution to the problem

$$
-\nabla \cdot(q \nabla G)=\delta\left(z-z_{0}\right),\left.\quad G\right|_{\partial \Omega_{0}}=0 .
$$

Let the exact image $u^{0}$ be a smooth function. We recall Greens second formula

$$
\begin{aligned}
& \int_{\Omega_{0}}\left(u^{0}(z) \nabla \cdot\left(q \nabla G\left(z_{0}, z\right)\right)-G\left(z_{0}, z\right) \nabla \cdot\left(q \nabla u^{0}(z)\right)\right) d z \\
& =\int_{\partial \Omega_{0}} q\left(u^{0}(z(s)) \frac{\partial G\left(z_{0}, z\right)}{\partial n}-G\left(z_{0}, z\right) \frac{\partial u^{0}(z(s))}{\partial n}\right) d s .
\end{aligned}
$$

Hence,

$$
\begin{gathered}
-u^{0}\left(z_{0}\right)-\int_{\Omega_{0}} G\left(z_{0}, z\right)\left(\nabla \cdot\left(q \nabla u^{0}(z)\right)\right) d z \\
=\int_{\partial \Omega_{0}} q u^{0}(z(s)) \frac{\partial G\left(z_{0}, z\right)}{\partial n} d s
\end{gathered}
$$

or

$$
\begin{aligned}
u^{0}\left(z_{0}\right)= & \int_{\Omega_{0}} G\left(z_{0}, z\right)\left(-\nabla \cdot\left(q \nabla u^{0}(z)\right)\right) d z \\
& +\int_{\partial \Omega_{0}} q u^{0}(z(s)) \frac{\partial\left(-G\left(z_{0}, z\right)\right)}{\partial n} d s
\end{aligned}
$$

Now by adding and subtracting the term $\int_{\Omega_{0}} G\left(z_{0}, z\right) f(z) d z$ from the right hand side of the equality above, we get

$$
\begin{aligned}
u^{0}\left(z_{0}\right)= & \int_{\Omega_{0}} G\left(z_{0}, z\right)\left(-\nabla \cdot\left(q \nabla u^{0}(z)\right)-f\right) d z \\
& +\int_{\Omega_{0}} G\left(z_{0}, z\right) f(z) d z+\int_{\partial \Omega_{0}} q u^{0}(z(s)) \frac{\partial\left(-G\left(z_{0}, z\right)\right)}{\partial n} d s
\end{aligned}
$$

Then, the function $u^{0}$ can be split in $u^{0}=u^{a}+u^{e}$, where

$$
u^{a}\left(z_{0}\right)=\int_{\Omega_{0}} G\left(z_{0}, z\right)\left(-\nabla \cdot\left(q \nabla u^{0}(z)\right)-f(z)\right) d z
$$


and

$$
u^{e}\left(z_{0}\right)=\int_{\Omega_{0}} G\left(z_{0}, z\right) f(z) d z+\int_{\partial \Omega_{0}} q u^{0}(z(s)) \frac{\partial\left(-G\left(z_{0}, z\right)\right)}{\partial n} d s .
$$

We inpaint the domain $\Omega_{0}$ by $u^{e}$ which satisfies

$$
-\nabla \cdot\left(q \nabla u^{e}\right)=f,\left.\quad u^{e}\right|_{\partial \Omega_{0}}=\left.u^{0}\right|_{\partial \Omega_{0}} .
$$

The component $u^{a}$ satisfies

$$
-\nabla \cdot\left(q \nabla u^{a}\right)=-\nabla \cdot\left(q \nabla u^{0}\right)-f,\left.\quad u^{a}\right|_{\partial \Omega_{0}}=0 .
$$

Then we are interested in the error

$$
\left\|u^{0}-u^{e}\right\|_{\infty}=\left\|u^{a}\right\|_{\infty} \leq M \int_{\partial \Omega_{0}} G\left(z_{0}, z\right) d z
$$

where $\left|\left(-\nabla \cdot\left(q \nabla u^{0}(z)\right)-f(z)\right)\right| \leq M$ for every $z \in \Omega_{0}$. Denote by $d$ the diameter of $\Omega_{0}$ and let $B_{d}$ be a disk centered at 0 with radius $d$. Then $\Omega_{0} \subset B_{d}$ and

$$
\int_{\Omega_{0}} G\left(z_{0}, z\right) d x d y \leq \int_{B_{d}} G\left(z_{0}, z\right) d x d y
$$

Now, to estimate the last integral in the inequality above we use the pointwise estimate of Green's function given in Theorem 7.1 in [24]. It says that there is a constant $K$ such that

$$
K^{-1} \hat{G}_{d} \leq G \leq K \hat{G}_{d}
$$

where $\hat{G}$ is the Greens function solving the Poisson equation on $B_{d}$ :

$$
-\Delta \hat{G}_{d}=\delta\left(z-z_{0}\right),\left.\quad \hat{G}_{d}\right|_{\partial B_{d}}=0 .
$$

Hence, the inequality in (7) becomes

$$
\int_{\Omega_{0}} G\left(z_{0}, z\right) d x d y \leq \int_{B_{d}} G\left(z_{0}, z\right) d x d y \leq K \int_{B_{d}} \hat{G}_{d}\left(z_{0}, z\right) d x d y .
$$

Furthermore, using the estimates in Theorem 1 in [23], we get

$$
\int_{\Omega_{0}} G\left(z_{0}, z\right) d x d y \leq \int_{B_{d}} G\left(z_{0}, z\right) d x d y \leq K \int_{B_{d}} \hat{G}_{d}\left(z_{0}, z\right) d x d y \leq K \frac{d^{2}}{4}
$$


This gives

$$
\left\|u^{0}-u^{e}\right\|_{\infty}=\left\|u^{a}\right\|_{\infty} \leq M \int_{\Omega_{0}} G\left(z_{0}, z\right) d z \leq M K \frac{d^{2}}{4} .
$$

Hence,

$$
|| u^{e}-\left.u^{0}\right|_{\Omega_{0}} \|_{\infty}=O\left(d^{2}\right)
$$

as $d \rightarrow 0$.

Our method allows for discontinuous $q$ while maintaining the same error estimation as for the harmonic inpainting. From the analysis we see that in the case when the coefficient $q$ is close to a constant then our method has the same error estimate as Harmonic inpainting. For the methods to differ the coefficient $q$ must be different in different regions of the inpainting domain, i.e. be close to zero along edges in the domain. In this case the error estimate is not entirely relevant and the constant $M$ quite large.

\section{Numerical results}

In this section we show numerical results intended to illustrate the potential of our method. For all tests we used images and the computations were carried out using Matlab. In the case of RGB color images we solve the inpainting problem for each color channel separately.
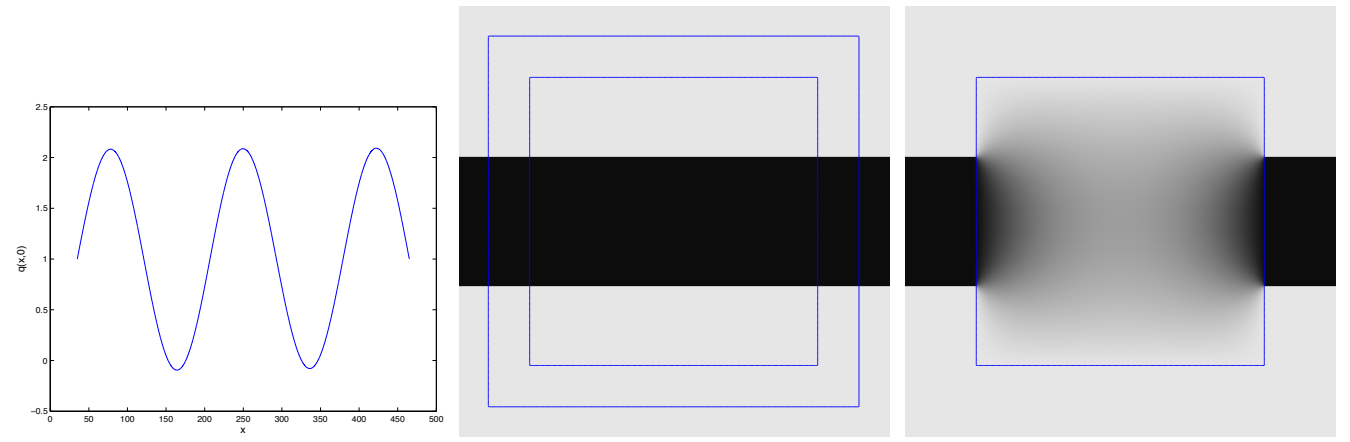

Figure 4: The coefficient $q(x, 0)$ we used for the inpainting (left) and the results obtained using coefficient based inpainting on an image that consists of a horizontal black stripe (middle) are displayed. The regions $\Omega_{0}$ and $\Omega_{1}$ are marked in the image. Also we present and the results obtained using Harmonic inpainting (right). The inpainting domain $\Omega_{0}$ is marked in the image. 
The errors in the reconstructions is measured using the Peak-Signal-ToNoise Ratio (PSNR), see e.g. [25], defined as

$$
\operatorname{PSNR}\left(I^{(1),} I^{(2)}\right)=10 \log _{10}\left(M A X^{2} / M S E\right), \quad M S E=\frac{1}{3 n_{1} n_{2}} \sum_{i, j}\left(I_{i, j}^{(1)}-I_{i, j}^{(2)}\right)^{2},
$$

where $I^{(1)}$ and $I^{(2)}$ are two color images of size $n_{1} \times n_{2}$ and $M A X$ is the dynamic range of the image, e.g. 255 for an 8-bit image.

For the first test we again used a $500 \times 500$ pixel image consisting of a single horizontal black stripe. Since the image doesn't contain any local maxima we used $f=0$ so the available information in the domain $\Omega_{1}$ was only used for finding a coefficient $q(x, y)$. For this experiment we used the model,

$$
q(x, y)=c_{1}+\sum_{k=2}^{n} c_{k} s_{k}(x), \quad s_{k}(x)=\sin (k \pi \theta(x)),
$$

where,

$$
\theta(x)=\frac{x-x_{\min }}{x_{\max }-x_{\min }},
$$

and $\Omega_{1} \subset\left[x_{\min }, x_{\max }\right] \times\left[y_{\min }, y_{\max }\right]$. For the test we used $n=10$ basis functions. The results are illustrated in Figure 4. The method works rather well. For this test the least squares problem used for determining $q(x, y)$ was under determined. The explanation is that the because of the specific test image that was chosen the only thing important is that $q(x, y)$ is close to zero along the horizontal edges of the black stripe. This can be achieved using only one of the sine components. Note that for this particular test the total variation scheme would also produce a close to perfect reconstruction. So a comparison is not needed.

For the second test we use a color photo of size $376 \times 351$ pixels. The inpainting problem is solved for each color channel separately. Here the main features are a set of vertical lines of discontinuity and hence we use basis functions oriented along the $y$-axis. For this particular test we represent the coefficient as

$$
q(x, y)=c_{1}+\sum_{k=2}^{n} c_{k} B_{k}(\theta(y)), \quad \theta(y)=\frac{y-y_{\min }}{y_{\max }-y_{\min }}
$$

where $B_{k}(x)$ are $B$-spline basis functions with support in the interval $(0,1)$. For this test the source term $f$ was set to zero. A total of $n=10$ basis 

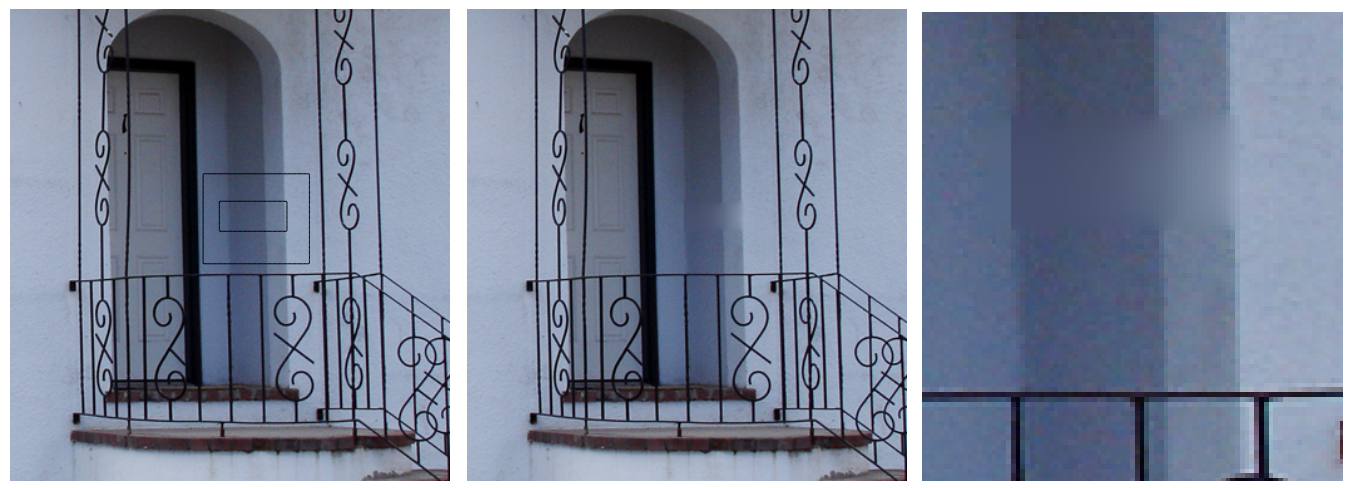

Figure 5: The original image and the inpainting domains (left) and the result after using our Coefficient based inpainting code (middle). We also display a close-up of the part of the image where the inpainting was performed (right). The difference between the original image and the inpainted one, given by the PSNR, is 50.3.

fucntions were used for this test. The results are presented in Figure 5. Also, the coefficient $q(x, y)$ and the basis functions are illustrated in Figure 6 . The coefficient $q(0, y)$ have clear minima that correspond to the left-most and right-most vertical line. Hence those are fairly sharp in the reconstruction. However due to the low number of basis functions used the minima are slightly missplaced. Hence the lines have moved in the reconstruction. This demonstrates that, while it can recreate sharp features in the image, the method is sensitive with respect to the choice of basis functions used. We also compute the PSNR in order to give an indication of the quality of the reconstruction.

As a third test we do a text removal experiment. A $360 \times 480$ pixel image has 14 letters written on it. The 6511 pixels hidden by the text are treated as unknown. The inpainting problem is solved for each letter separately. For each letter we let $\Omega_{1} \cup \Omega_{0}$ be a small rectangle that contains the letter. The information in $\Omega_{1}$ is used for finding both $q(x, y)$ and $f(x, y)$. The basis functions are of the type,

$$
s_{k, l}(x, y)=\sin (k \pi \theta(x)) \sin (l \pi \eta(y)),
$$

where,

$$
\theta(x)=\frac{x-x_{\min }}{x_{\max }-x_{\min }}, \quad \eta(y)=\frac{y-y_{\min }}{y_{\max }-y_{\min }} .
$$

In addition to $s_{1,1}(x, y)=1$ a total of 14 basis functions, with frequencies 

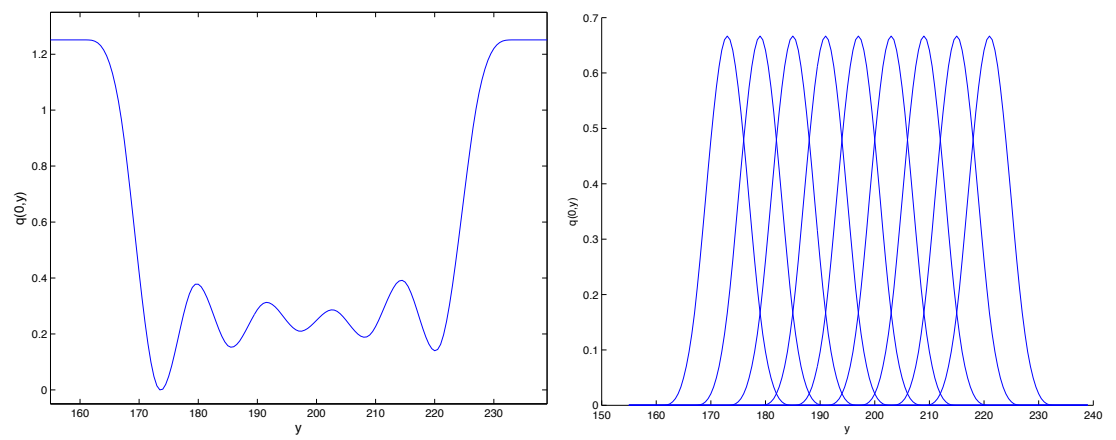

Figure 6: The coefficient $q(x, y)=q(0, y)$ used for the test (left). Also the $9 B$-spline basis functions that were used for representing the coefficient (right). Here the pixels 154-242 are inside the outer box $\Omega_{1}$.
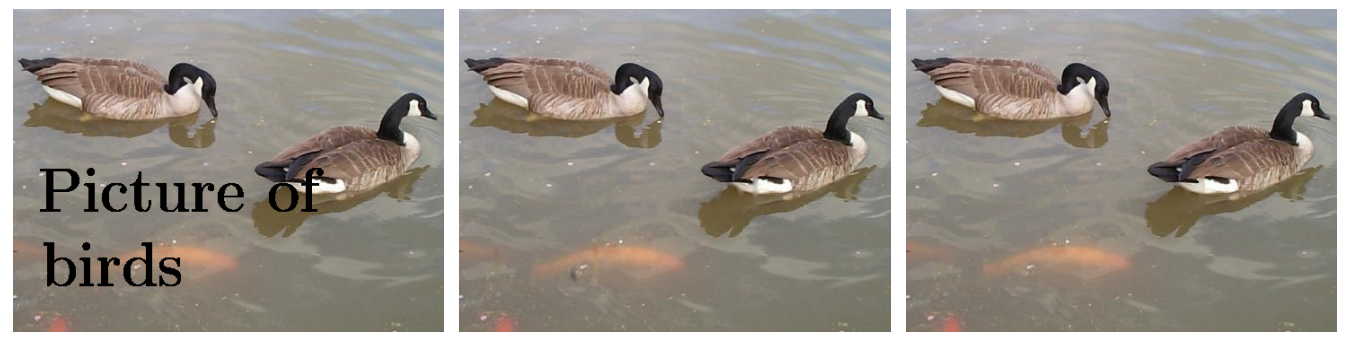

Figure 7: The image with text added (left) and the result after performing coefficient based inpainting on each of the 14 letters (middle). For for comparison we also display the results obtained using Total Variation inpainting (right).

$1 \leq k+l \leq 4$, were used. Here $f=0$ was used. The result is presented in Figure 7. The result is fairly good. The water surface is very accurately reconstructed. As a comparison we also display the results obtained from using the Total Variation inpainting scheme. In this case both methods reach a PSNR value of around 19.5 when compared to the original image. The results obtained using Total Variation are slightly better but, as mentioned, the computational cost is significantly higher as the method is non-linear and the algorithm iterative.

As a fourth experiment we take a photo $256 \times 384$ with scratches on it. For this test there are four different damaged regions with interesting features to reconstruct. The inpainting problem is solved for each damaged region separately. We use the same $n=15$ basis functions as in Test 3 above, but 
now a non-zero source term $f$ was used. The original image and the regions that are used for finding the optimal coefficient is displayed in Figure 8. Also the reconstructed image obtained using our method and the image obtained by the Total Variation (TV) method is displayed. Also we give a close-up of two of the damaged areas in Figure 9. For the experiments we computed

$$
P S N R\left(I_{\text {orig }}, I_{\text {coef }}\right)=25.8, \quad \text { and } P S N R\left(I_{\text {orig }}, I_{t v}\right)=25.7
$$

For this case our method is competitive with the more computationally demanding total variation method. Though we emphasize that this case is close to ideal for both methods with very thin regions to inpaint and also no very sharp features that needs to be reconstructed. Hence both methods perform very well.

\section{Concluding Remarks}

In this paper, we have shown that parameter identification problems can be applied to image inpainting. Our inspiration has been the Laplace equation which gives raise to the simple harmonic inpainting scheme. In order to get better results than the harmonic inpainting, we have studied an elliptic equation in divergence form with a diffusion coefficient $q$ and source term $f$. To fill in the region of missing data with data from the surrounding area, we assume that the diffusion coefficient $q$ and the source term $f$ are unknown and have to be estimated from a region where the pixel information is known. This is an inverse problem. Numerical results shows that the method works fairly well. Both in comparison to harmonic inpainting and the total variation method. It is clear that our method is very sensitive with
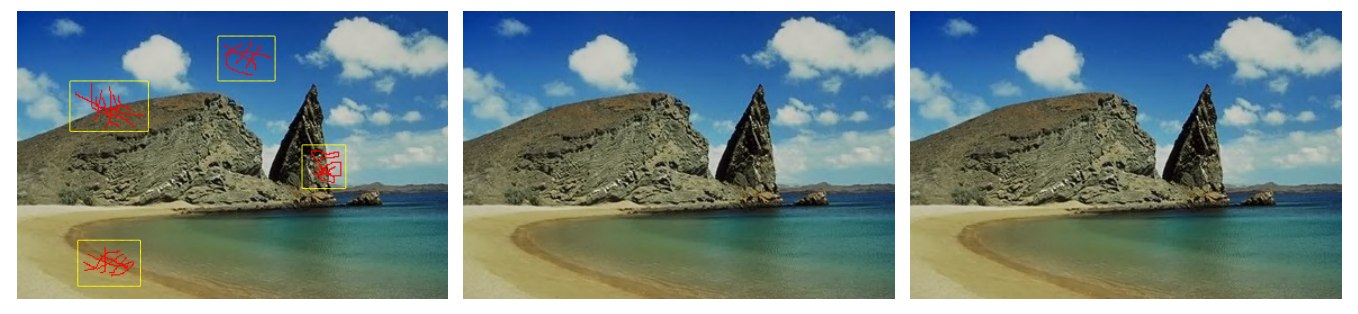

Figure 8: The original image with damaged areas (left) and the reconstructions obtained by using the coefficient based approach (middle) and by using the Total Variation method (right). 

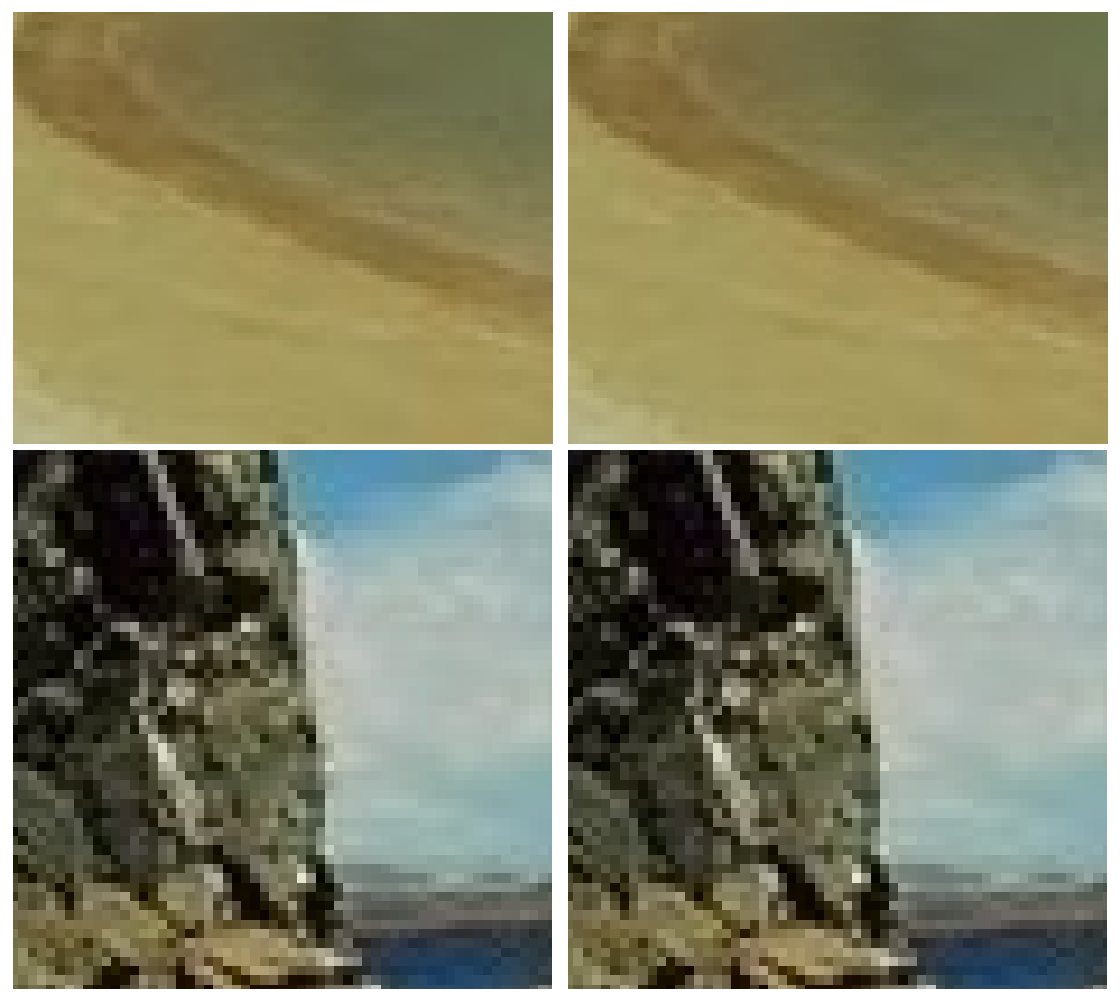

Figure 9: A close-up of two of the damaged areas. We display the results obtained using the coefficient based method (left) and those obtained using Total Variation inpainting (right).

respect to the basis fucntions used to represent coefficients $q$ and $f$. Methods for chosing the appropriate basis functions for a concrete inpainting problem is something that needs to be explored further. Also since a limited number of basis functions is used for representing the coefficients $q$ and $f$ we can't hope to reconstruct complicated shapes for lines of discontinuity inside the domain.

The method retains the advantages of the simple harmonic inpainting technique. In our method we use basis functions that are defined globally on the whole domain $\Omega_{1}$ to specify the diffusion coefficient. We compute the diffusion coefficient, using the known image information, once; before the inpainting takes place. This means that our scheme does not require iteration. An alternative is to use local information in the known part of 
the image to estimate the diffusion coefficient locally; and then propagate this information into the inpainting domain along geometric curves. This is something we intend to do in the future.

\section{References}

[1] C. Guillemot, O. Le Meur, Image Inpainting: Overview and Recent Advances, IEEE Signal Processing Magazine, vol. 31, no. 1, pp. 127$144,2014$.

[2] A. A. Efros, T. K. Leung, Texture synthesis by non-parametric sampling, in: IEEE International Conference on Computer Vision, Corfu, Greece, 1999, pp. 1033-1038.

[3] D. Mumford, J. Shah, Optimal approximations by piecewise smooth functions and associated variational problems, Comm. Pure Appl. Math. 42 (5) (1989) 577-685.

[4] M. Nitzberg, D. Mumford, T. Shiota, Filtering, segmentation and depth, Vol. 662 of Lecture Notes in Computer Science, Springer-Verlag, Berlin, 1993.

[5] S. Masnou, J.-M. Morel, Level lines based disocclusion, in: Proc. of IEEE ICIP 1998, 1998.

[6] M. Bertalmio, G. Sapiro, V. Caselles, C. Ballester, Image inpainting, Computer Graphics, SIGGRAPH 2000 (2000) 355-362.

[7] M. Bertalmio, A. Bertozzi, G. Sapiro, Navier-stokes, fluid dynamics, and image and video inpainting, in: Proc. IEEE Computer Vision and Pattern Recognition(CVPR), 2001, pp. 355-362.

[8] M. Burger, H. Lin, C.-B. Schnlieb, Cahn-hilliard inpainting and a generalization for grayvalue images, SIAM J. Imag. Sci. 3.

[9] H. Grossauer, O. Scherzer, Using the complex ginzburg-landau equation for digital inpainting in $2 \mathrm{~d}$ and $3 \mathrm{~d}$, in: In Scale Space Methods in Computer Vision, Springer, 2003.

[10] J.-F. Aujol, S. Ladjal, S. Masnou, Exemplar-based inpainting from a variational point of view, SIAM J. Math. Anal. 42 (3) (2010) 1246-1285. 
[11] P. Arias, G. Facciolo, V. Caselles, G. Sapiro, A variational framework for exemplar-based image inpainting, International Journal of Computer Vision 93 (2011) 319-347.

[12] Y. Liu, V. Caselles, Exemplar-Based Image Inpainting Using Multiscale Graph Cuts, IEEE Transactions on Image Processing, vol. 22, no. 5, pp. 1699-1711, 2013.

[13] L. I. Rudin, S. Osher, E. Fatemi, Nonlinear total variation based noise removal algorithms, Physica D 60 (1-4) (1992) 259-268.

[14] T. F. Chan, S. J., Non-texture inpainting by curvature driven diffusions (cdd), J. Visual Comm. Image Rep. 12 (8) (2003) 882-889.

[15] T. F. Chan, S. H. Kang, J. Shen, Euler's elastica and curvature-based inpainting, SIAM J. Appl. Math. 63 (2) (2002) 564-592 (electronic).

[16] D. Tschumperlé, Fast anisotropic smoothing of multi-valued images using curvature-preserving pde's., International Journal of Computer Vision 68 (1) (2006) $65-82$.

[17] F. Bornemann, T. Mrz, Fast image inpainting based on coherence transport, Journal of Mathematical Imaging and Vision 28 (2007) 259-278.

[18] C. Qin, F. Cao, X. P. Zhang, Efficient Image Inpainting Using Adaptive Edge-Preserving Propagation, The Imaging Science Journal, vol. 59, no. 4, pp. 211-218, 2011.

[19] R. Chen, X. Li, S. Li, Image inpainting based on anisotropic mrf model, in: Proc. of SPIE, Vol. 7498, SPIE, 2009, pp. 749-846.

[20] C. Qin, S. Wang, X. Zhang, Simultaneous inpainting for image structure and texture using anisotropic heat transfer model, Multimedia Tools and Applications, vol. 56, no. 3, pp. 469-483, 2012.

[21] T. F. Chan, J. Shen, Image processing and analysis, Society for Industrial and Applied Mathematics (SIAM), Philadelphia, PA, 2005, variational, PDE, wavelet, and stochastic methods.

[22] D. Gilbarg, N. S. Trudinger, Elliptic partial differential equations of second order, Classics in Mathematics, Springer-Verlag, Berlin, 2001. 
[23] T. F. Chan, S. H. Kang, Error analysis for image inpainting, J. Math. Imaging Vision 26 (1-2) (2006) 85-103.

[24] W. Littman, G. Stampacchia, H. F. Weinberger, Regular points for elliptic equations with discontinuous coefficients, Annali Della Scoula Normale Superiore Di Pisa, Classe di Scinze $3^{e}$ srie, tome 17 (1-2) (1963) $43-77$.

[25] Q. Huynh-Thu, M. Ghanbari, Scope of validity of psnr in image/video quality assessment, Electronics Letters 44 (13) (2008) 800-801. 